\title{
Standard versus trans-epithelial collagen cross-linking in keratoconus patients suitable for standard collagen cross-linking
}

This article was published in the following Dove Press journal:

Clinical Ophthalmology

18 March 2015

Number of times this article has been viewed

S Rossi

A Orrico

C Santamaria

$\checkmark$ Romano

L De Rosa

F Simonelli

G De Rosa

Multidisciplinary Department of Medical, Surgical and Dental Sciences, Eye Clinic, Second University of Naples, Naples, Italy
Purpose: Evaluating the clinical results of trans-epithelial collagen cross-linking (CXL) and standard CXL in patients with progressive keratoconus.

Methods: This prospective study comprised 20 eyes of 20 patients with progressive keratoconus. Ten eyes were treated by standard CXL and ten by trans-epithelial cross-linking (TE-CXL, epithelium on) with 1 year of follow-up. All patients underwent complete ophthalmologic testing that included pre- and postoperative uncorrected visual acuity, corrected visual acuity, spherical error, spherical equivalent, corneal astigmatism, simulated maximum, minimum, and average keratometry, coma and spherical aberration, optical pachymetry, and endothelial cell density. Intraand postoperative complications were recorded. The solution used for standard CXL comprised riboflavin $0.1 \%$ and dextran $20.0 \%$ (Ricrolin), while the solution for TE-CXL (Ricrolin, TE) comprised riboflavin $0.1 \%$, dextran $15.0 \%$, trometamol (Tris), and ethylenediaminetetraacetic acid. Ultraviolet-A treatment was performed with UV-X System at $3 \mathrm{~mW} / \mathrm{cm}^{2}$.

Results: In both the standard CXL group (ten patients, ten eyes; mean age, $30.4 \pm 7.3$ years) and the TE-CXL group (ten patients, ten eyes; mean age, $28 \pm 3.8$ years), uncorrected visual acuity and corrected visual acuity improved significantly after treatment. Furthermore, a significant improvement in topographic outcomes, spherical error, and spherical equivalent was observed in both groups at month 12 posttreatment. No significant variations were recorded in other parameters. No complications were noted.

Conclusion: A 1-year follow-up showed stability of clinical and refractive outcomes after standard CXL and TE-CXL.

Keywords: corneal disease, corneal thickness, keratoconus, refractive outcomes, surgical technique

\section{Introduction}

Keratoconus is a degenerative, bilateral, asymmetrical, non-inflammatory disease, which induces biomechanical corneal weakening. ${ }^{1,2}$ Two different variants of the same surgical technique are adopted for treatment of this disease. Both methods, standard cross-linking (CXL, epi-off CXL) and trans-epithelial cross-linking (epi-on CXL), use ultraviolet-A (UVA) light and riboflavin (photosensitizer, vitamin B2). The photochemical reaction between the latter leads to the development of chemical bonds between collagen fibrils within the corneal stroma thereby strengthening the cornea and slowing or stopping the progression of keratoconus and other corneal ectasia (ie, post-laser in situ keratomileusis and pellucid marginal degeneration). ${ }^{3-7}$ Epi-off CXL involves epithelium debridement. Long-term clinical studies have shown that this method slows and in most cases blocks keratoconus progression ${ }^{8-11}$ and in some cases, it also improves refractive and topographic features. ${ }^{7,12}$ Epi-on CXL is a 
technique more recently introduced and is performed without epithelial debridement. This technique aims to reduce postoperative pain and risk of infection, eliminates the need of an operating room, and ensures disease stability. ${ }^{13}$ In terms of efficacy of both techniques, epi-on CXL and epi-off CXL, opinions seem to be controversial in this regard. ${ }^{14}$ Literature reports studies that confirm the safety of both techniques. ${ }^{15-18}$ These works found the procedures to be safe to the endothelium, confirming in vivo the sparing of corneal nerve fibers seen in vitro. ${ }^{19,20}$ However, further studies showed that the apoptotic effect of epi-on irradiation ${ }^{18,21}$ was concentrated at a very superficial level, being limited to the anterior stroma and that riboflavin penetration was not homogeneous with the epithelium in situ using the same UVA power, as for standard treatment $\left(3 \mathrm{~mW} / \mathrm{cm}^{2}\right){ }^{21,22}$

In most previous works, the epi-on technique was performed on patients with central corneal thickness (CCT) greater than $400 \mu \mathrm{m}$ and homogeneous topographic and anatomical parameters (in a defined range of thickness and keratometry). ${ }^{13,23,24}$ The aim of our study was to evaluate the efficacy of the two treatments in two homogeneous groups (for age, anatomical and topographic parameters) of patients with progressive keratoconus, in order to better define the validity of both techniques.

\section{Methods}

Patients with progressive keratoconus were recruited at the Eye Clinic of the Second University of Naples, Italy, from May 2012 to July 2012. Twenty eyes from 20 affected patients were included in the study. Patients were randomly assigned to one of the two treatment groups (ten eyes were treated with epi-off CXL, and the other ten eyes were treated with epi-on CXL). The study adhered to the tenets of the Declaration of Helsinki and was approved by a departmental Institutional Review Board at the University Hospital Second University of Naples. Moreover, each patient gave written informed consent for his or her involvement. All patients included in the treatment protocol were of age greater than 18 years and presented progressive keratoconus with a documented clinical and instrumental (topographic, pachymetric, or aberrometric) worsening in the previous 6 months of observation. The parameters defined to establish keratoconus progression and inclusion criteria were worsening of uncorrected distance visual acuity (UDVA) and/or corrected distance visual acuity (CDVA) of more than one Snellen line, an increase in central corneal astigmatism of at least $1.00 \mathrm{D}$, an increase in the maximum cone apex curvature of at least $1.00 \mathrm{D}$, a reduction of at least $10 \mu \mathrm{m}$ or more in the thinnest point and the thinnest point being no lower than $400 \mathrm{~m}$, a clear cornea on slit lamp, and the absence of Vogt striae. Exclusion criteria were any coexisting ocular disease or corneal opacities possibly affecting visual acuity, previous intraocular surgery, history of herpetic keratitis, severe dry eye, and concomitant autoimmune diseases.

Clinical pre- and postoperative testing included UDVA, CDVA, slit-lamp exam, spherical error, spherical equivalent (SE), corneal astigmatism, simulated maximum, minimum, and average keratometry $(K)$, coma and spherical aberration, CCT, and endothelial cell density (ECD). All intra- and postoperative adverse events were recorded, and complete testing was repeated at three and 12 posttreatment time points, except spherical aberration, coma aberration, and root mean sphere.

UDVA and CDVA were recorded using a LogMAR Early Treatment Diabetic Retinopathy Study chart at $4 \mathrm{~m}$. Pentacam topography (Oculus, Wetzlar, Germany) was used to evaluate pre- and postoperative corneal topography and pachymetry. Corneal endothelium was photographed using a noncontact specular microscope NonconRobo Sp-8000 (Konan), and ECD was calculated after marking at least 40 cells. Coma and spherical aberration and root mean squares were calculated using Eye Top (Costruzione Strumenti Oftalmici, Florence, Italy). Contact lens wearers were instructed to discontinue use of spherical soft lenses for a minimum of 4 weeks and soft toric, rigid gas-permeable and hard lenses for a minimum of 12 weeks before the preoperative eye examination. Epi-off CXL technique was performed under topical anesthesia instilling 4\% lidocaine and $1.0 \%$ pilocarpine. Mechanical corneal epithelium removal over $9.0 \mathrm{~mm}$ was performed. Riboflavin (0.1\% in 20\% dextran solution; Ricrolin; Sooft, Montegiorgio, Italy) was administered topically every 2 minutes for 30 minutes and was continued every 2 minutes during UVA exposure. The cornea was exposed to UVA $370 \mathrm{~nm}$ light (UV-X System; Peschke Meditrade GmbH, Hünenberg, Switzerland) for 30 minutes at an irradiance of $3.0 \mathrm{~mW} / \mathrm{cm}^{2}$. Ofloxacin and cyclopentolate drops were administered, and therapeutic contact lens (LAC ACUVUE-etafilcon A) were applied for 3 days after surgery. Patients were discharged with topical tobramycin to apply four times a day for 1 week, dexamethasone phosphate $0.1 \%$ four times a day for 2 weeks, then tapering to zero, and lubricating eye drops to use for the following 3 months. The epi-on CXL group was treated using following technique: corneal epithelial removal was not performed, and corneal imbibition was obtained with $0.1 \%$ riboflavin- $15 \%$ dextran solution supplemented with Tris-hydroxymethylaminomethane and sodium ethylenediaminetetraacetic acid (Ricrolin TE; Sooft) by instillation 
of two drops every 5 minutes for 30 minutes at $3.0 \mathrm{~mW} /$ $\mathrm{cm}^{2}$. One drop of $1 \%$ pilocarpine was administered $30 \mathrm{~min}$ utes before treatment. Twenty minutes before UV radiation, the cornea was anesthetized with single-dose anesthetic eye drops (4\% lidocaine). Postoperative corticosteroid drops were not instilled. All patients were operated by same surgeon.

A sample size of ten eyes was required to detect a difference of $0.75 \mathrm{D}$ between the $K_{\max } 12$ months after treatment and at baseline, at a significance level of 0.05 and a power of $80 \%$, assuming a standard deviation of $0.75 \mathrm{D}$. Data are expressed as mean \pm standard deviation. Statistical analysis was performed by using MATLAB r2014a (MathWorks, Natick, MA, USA). In order to deal with non-normality of some data, differences between the two groups (at baseline and in treatment effect) were assessed using Wilcoxon rank sum test, while differences over the follow-up time points were evaluated by using Wilcoxon signed rank test with Bonferroni correction for multiple comparisons. A $P$-value $<0.05$ was considered statistically significant.

\section{Results}

The study sample consisted of 20 eyes of 20 patients. Ten eyes were treated with epi-off CXL (group A), and the other ten eyes were treated with epi-on CXL (group B) (Table 1). No significant differences in age and pachymetric and keratometric parameters between the two groups were assessed at baseline, while UDVA and CDVA were higher in the epi-off CXL group.

In group $\mathrm{A}$, mean age was $30.4 \pm 7.3$ years (range: 22-42 years). Mean baseline UDVA was 0.65 LogMAR

Table I Outcomes before and after epithelium-off CXL and TE-CXL

\begin{tabular}{|c|c|c|c|c|c|}
\hline & \multirow[t]{2}{*}{ Baseline } & \multirow[t]{2}{*}{3 months } & \multirow[t]{2}{*}{12 months } & \multicolumn{2}{|l|}{$P$-value } \\
\hline & & & & $\begin{array}{l}3 \text { months } \\
\text { versus baseline }\end{array}$ & $\begin{array}{l}12 \text { months } \\
\text { versus baseline }\end{array}$ \\
\hline \multicolumn{6}{|l|}{ Epithelium-off $C X L, n=10$ eyes, 5 M/5 F } \\
\hline Uncorrected visual acuity, LogMAR & $0.65(0.15)^{* *}$ & $0.64(0.09)$ & $0.49(0.03)$ & 0.846 & 0.002 \\
\hline Corrected visual acuity, LogMAR & $0.22(0.04)^{* *}$ & $0.17(0.04)$ & $0.08(0.02)$ & 0.002 & 0.002 \\
\hline Spherical error (D) & $4.2(1.68)$ & $4.65(1.74)$ & $3.77(1.63)$ & 0.037 & 0.002 \\
\hline Spherical equivalent & $6.48(2.62)$ & $7.09(2.63)$ & $5.92(2.55)$ & 0.037 & 0.014 \\
\hline$K_{\min }(D)$ & $46.97(4.74)$ & $47.06(4.82)$ & $46.49(4.65)$ & 0.846 & 0.014 \\
\hline$K_{\max }(\mathrm{D})$ & $51.64(6.8 I)$ & $51.89(6.68)$ & $50.8 \mathrm{I}(6.5 \mathrm{I})$ & 0.922 & 0.010 \\
\hline Mean $K(D)$ & $49.31(5.72)$ & $49.47(5.7)$ & $48.66(5.5 \mathrm{I})$ & 0.846 & 0.010 \\
\hline Topographic astigmatism & $4.67(2.55)$ & $4.83(2.38)$ & $4.31(2.4)$ & 0.160 & 0.010 \\
\hline Apex keratometry (D) & $57.68(5.96)$ & $57.8(5.83)$ & $56.88(5.91)$ & 1.000 & 0.014 \\
\hline Inferior-superior symmetry index $\left(\mathrm{mm}^{2}\right)$ & $6.2(3.64)$ & $6.15(3.61)$ & $6(3.57)$ & 1.000 & 0.695 \\
\hline Spherical aberration, Z4 & $0.297(0.13)$ & & $0.302(0.18)$ & & 0.375 \\
\hline Coma aberration, Z3 & $3.544(1.4)$ & & $3.165(1.23)$ & & 0.064 \\
\hline Root mean sphere & $7.254(1.98)$ & & $6.156(1.99)$ & & 0.002 \\
\hline Endothelial cell density (cell/mm²) & $2,478.6(64.9)^{*}$ & $2,474(65.3)$ & $2,447.2(68.2)$ & 0.275 & 0.084 \\
\hline Central corneal thickness $(\mu \mathrm{m})$ & $454(14.42)$ & $462.5(14.79)$ & $452.2(14.65)$ & 0.160 & 0.492 \\
\hline \multicolumn{6}{|l|}{ TE-CXL, $n=10$ eyes, 6 M/4 F } \\
\hline Uncorrected visual acuity, LogMAR & $0.88(0.19)$ & $0.87(0.18)$ & $0.75(0.19)$ & 0.695 & 0.002 \\
\hline Corrected visual acuity, LogMAR & $0.36(0.13)$ & $0.37(0.12)$ & $0.21(0.1 \mathrm{I})$ & 0.375 & 0.002 \\
\hline Spherical error (D) & $3.1(2.95)$ & $3.45(3.02)$ & $2.65(2.55)$ & 0.006 & 0.004 \\
\hline Spherical equivalent & $5.16(3.08)$ & $5.6(3.13)$ & $4.3(2.8)$ & 0.160 & 0.010 \\
\hline$K_{\min }(D)$ & $47.7(4.74)$ & $47.03(3.93)$ & $46.56(4.39)$ & 0.922 & 0.010 \\
\hline$K_{\max }(D)$ & $52.4 I(5.39)$ & $51.56(4.9)$ & $50.5(5.37)$ & 0.275 & 0.010 \\
\hline Mean $K(D)$ & $50.05(4.97)$ & $49.27(4.36)$ & $48.5(4.82)$ & 0.557 & 0.014 \\
\hline Topographic astigmatism & $4.7(2.01)$ & $4.53(1.93)$ & $3.84(1.95)$ & 0.193 & 0.010 \\
\hline Apex keratometry $(D)$ & $57.45(5.19)$ & $55.89(5.14)$ & $54.89(4.88)$ & 0.002 & 0.002 \\
\hline Inferior-superior symmetry index $\left(\mathrm{mm}^{2}\right)$ & $8.47(1.92)$ & $8.25(1.7)$ & $7.29(1.69)$ & 0.432 & 0.002 \\
\hline Spherical aberration, Z4 & $0.317(0.21)$ & & $0.28(0.19)$ & & 0.846 \\
\hline Coma aberration, Z3 & $4.26(1.78)$ & & $3.51(1.34)$ & & 0.557 \\
\hline Root mean sphere & $6.60(2.54)$ & & $5.43(1.77)$ & & 0.002 \\
\hline Endothelial cell density (cell/mm²) & $2,325(205.3)$ & $2,290(210.2)$ & $2,272(199.4)$ & 0.160 & 0.084 \\
\hline Central corneal thickness $(\mu \mathrm{m})$ & $45 I . I(39.5 I)$ & $452.5(38.31)$ & $448.4(37.32)$ & 0.695 & 0.625 \\
\hline
\end{tabular}

Notes: The data are expressed as mean (standard deviation). Significant $P$-values are given in bold (ie, lower than 0.017 , according to Bonferroni correction for multiple comparisons). *Statistically significant difference between the two groups at baseline $(P<0.05)$; $* *$ Statistically significant difference between the two groups at baseline $(P<0.00 \mathrm{I})$. Abbreviations: CXL, cross-linking; TE-CXL, trans-epithelial cross-linking; $M$, male; F, female; D, diopter; $K$, keratometry. 

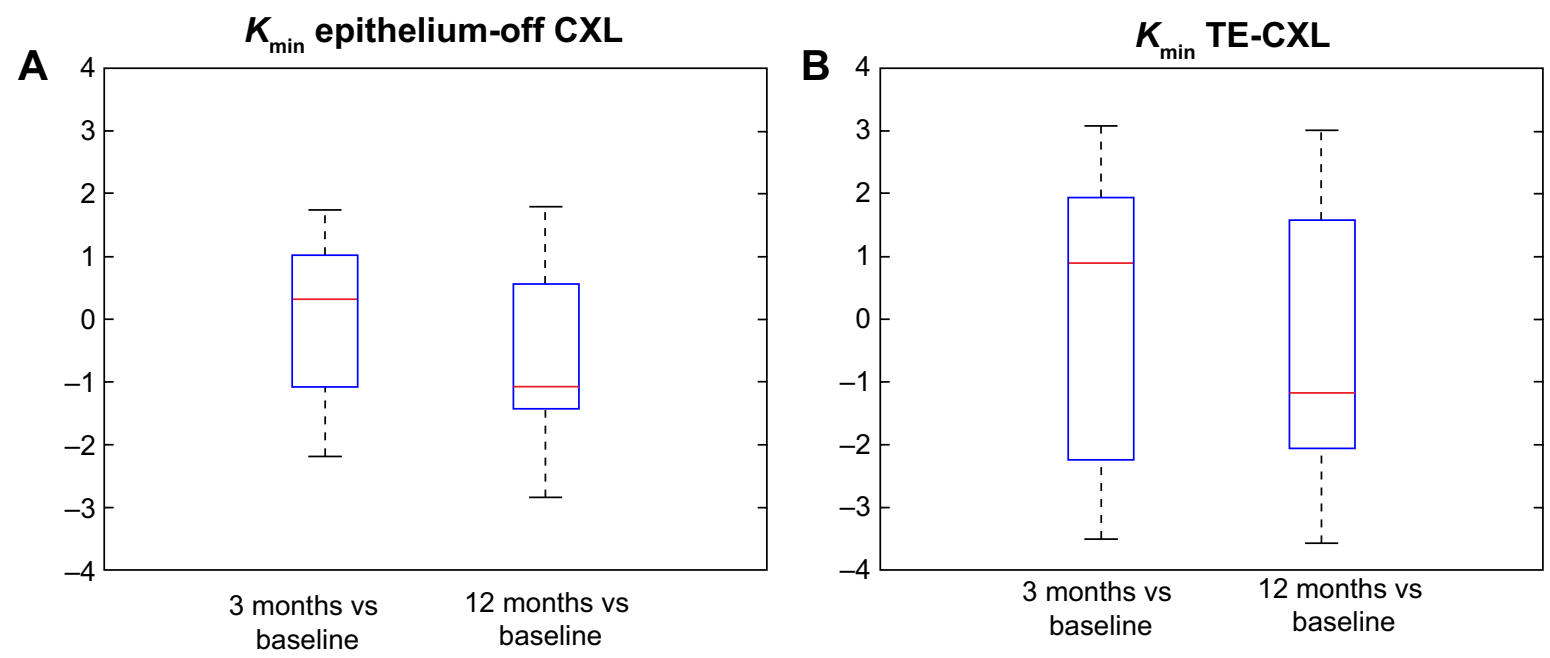

Figure I The simulated minimum keratometry $(K)$ after epithelium-off $C X L(A)$ and after epithelium-on $C X L(B)$.

Abbreviation: CXL, cross-linking.

(SD \pm 0.15 LogMAR). UDVA improvement (mean 0.64 LogMAR; SD \pm 0.09 LogMAR) observed at the 3-month postoperative time point became statistically significant $(P<0.05)$ at the 12-month time point with median UDVA $=0.49$ LogMAR (SD \pm 0.03 LogMAR). Mean baseline CDVA was 0.22 LogMAR ( $\mathrm{SD} \pm 0.04 \mathrm{LogMAR}$ ), and this value started to increase significantly from the 3 -month postoperative time point $(0.17$ LogMAR; SD \pm 0.04 LogMAR; $P<0.05$ ). All topographic parameters $\left(K_{\min }, K_{\max }\right.$, and mean $K$ ) showed a statistically significant improvement at 12 months post-surgery. The improvement in $K_{\min }$ and $K_{\max }$ over the follow-up is shown in Figures $1 \mathrm{~A}$ and $2 \mathrm{~A}$, respectively.

In group $\mathrm{B}$, mean age was $28 \pm 3.8$ years (range: 24-34 years). Median baseline UDVA was 0.88 LogMAR (SD \pm 0.19 LogMAR). UDVA improvement (median 0.87

A

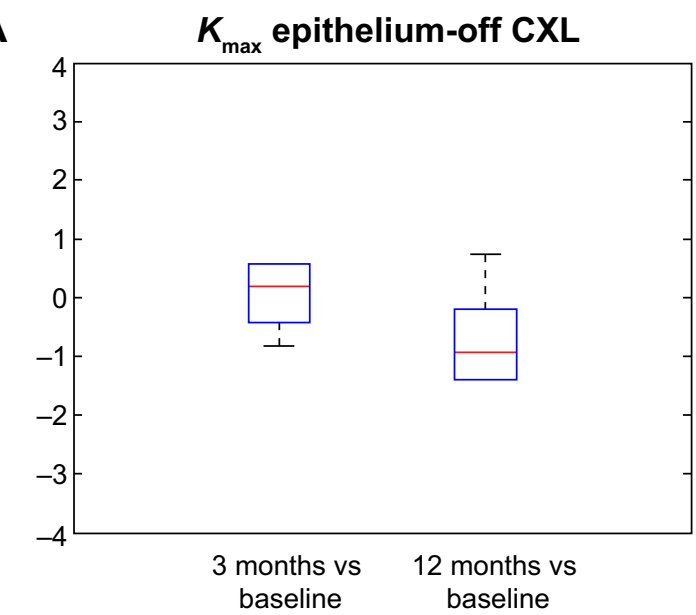

LogMAR; SD \pm 0.18 LogMAR) recorded at 3 months became statistically significant $(P<0.05)$ at 12 months post-surgery, when median UDVA was 0.75 LogMAR (SD \pm 0.19 LogMAR). Median baseline CDVA was 0.36 LogMAR (SD \pm 0.13 LogMAR) and increased significantly 12 months post-surgery (0.21 LogMAR; SD \pm 0.11 LogMAR; $P<0.05)$. At the same time point, a statistically significant improvement was also recorded in topographic parameters $\left(K_{\text {min }}, K_{\max }\right.$, and mean $K$ ) (Figures 1B and 2B), spherical error, $\mathrm{SE}$, topographic astigmatism, apex keratometry, inferiorsuperior symmetry index, root mean sphere. CCT, ECD, coma aberration, and spherical aberration remained stable up to 12 months after treatment $(P>0.05)$.

The treatment effect on the main clinical features between the two groups is compared in Table 2. No significant

B

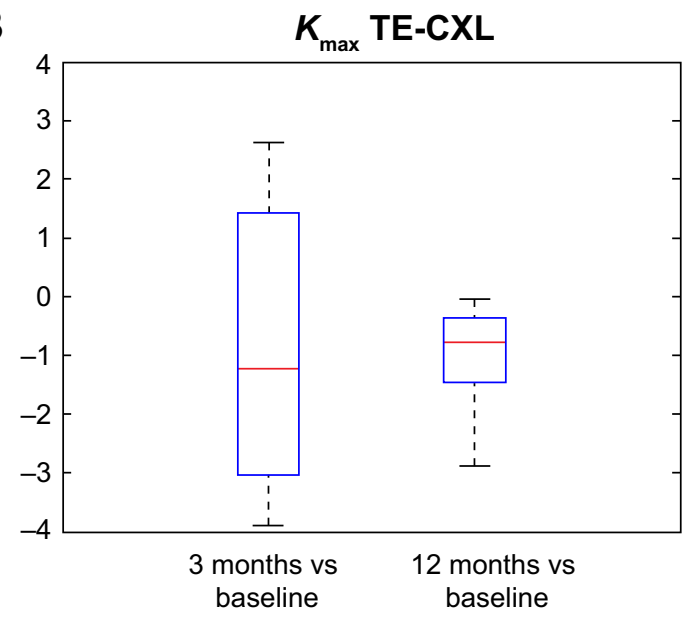

Figure 2 The simulated maximum keratometry $(K)$ after epithelium-off $C X L(\mathbf{A})$ and after epithelium-on CXL (B). Abbreviation: CXL, cross-linking. 
Table 2 Comparison of treatment effects between the two groups (epithelium-off CXL and TE-CXL)

\begin{tabular}{|c|c|c|c|c|c|}
\hline & \multicolumn{2}{|c|}{$\begin{array}{l}\text { Epithelium- } \\
\text { off CXL }\end{array}$} & \multicolumn{2}{|c|}{ TE-CXL } & \multirow[t]{2}{*}{$P$-value } \\
\hline & Mean & SD & Mean & SD & \\
\hline Uncorrected visual acuity, LogMAR & -0.15 & 0.07 & -0.12 & 0.06 & 0.38 \\
\hline Corrected visual acuity, LogMAR & -0.09 & 0.03 & -0.16 & 0.05 & 0.003 \\
\hline Spherical error (D) & -0.88 & 0.46 & -0.88 & 0.57 & 0.97 \\
\hline Spherical equivalent & -1.17 & 0.43 & -1.17 & 0.56 & 0.97 \\
\hline$K_{\min }(D)$ & -0.57 & 1.43 & -0.57 & 2.09 & 0.79 \\
\hline$K_{\max }(D)$ & -1.08 & 2.08 & -1.06 & 1.00 & 0.97 \\
\hline Mean $K(D)$ & -0.81 & 2.08 & -0.81 & 1.76 & 0.85 \\
\hline Topographic astigmatism & -0.52 & 0.29 & -0.69 & 0.91 & 0.79 \\
\hline Apex keratometry (D) & -0.92 & 1.46 & -1.00 & 1.42 & 0.85 \\
\hline Root mean sphere & 6.16 & 1.04 & 5.43 & 0.81 & 0.12 \\
\hline
\end{tabular}

Note: Significant $P$-values are given in bold.

Abbreviations: $C X L$, cross-linking; $T E-C X L$, trans-epithelial cross-linking; SD standard deviation; $\mathrm{D}$, diopter; $K$, keratometry.

differences were observed between the groups A and B, with only exception of a higher improvement in CDVA in group B.

No patients were lost at follow-up. During follow-up, no ocular or systemic adverse events were observed. No corneal edema, no haze, and no re-epithelialization delay were noticed.

\section{Discussion}

In this study, we analyzed refractive and functional outcomes after epi-off CXL and epi-on CXL in two groups of patients with progressive keratoconus. In order to assess the effectiveness of the two treatments, we removed all the confounding factors related to the patients, using the two techniques in two groups of patients with homogeneous characteristics at baseline. In fact, all eyes showed a CCT greater than $400 \mu \mathrm{m}$ and homogeneous topographic and anatomical parameters (in a defined range of thickness and keratometry). ${ }^{13,23,24}$ The aim of our study was to evaluate the efficacy of the two treatments in two homogeneous groups. Patients were randomly assigned to one of the two treatment groups. At 12 months posttreatment, our results showed that both procedures proved to be useful and effective in halting the progression of keratoconus, in accordance with previous reports. ${ }^{13,21,24-26}$ Furthermore, the results obtained using both procedures showed reduced mean SE and spherical error, improvement in UDVA and CDVA, and reduced topographic power after 12 months. Apex keratometry improvement highlighted the flattening of the corneal cone, as in other studies. ${ }^{7,10,25}$ No significant variations were recorded in spherical aberration and coma aberration, and CCT remained constant in both groups at 12 months post-surgery. Vinciguerra et al pointed out a significant decrease in corneal pachymetry at 12 months, but this prospective study treated patients with advanced keratoconus. ${ }^{27}$ The two most recent studies that analyze the effectiveness of epi-on CXL and epi-off CXL were performed by Caporossi et al ${ }^{24}$ and Magli et al. ${ }^{11}$ Caporossi et al ${ }^{24}$ reported a study of 26 eyes treated by epi-on CXL, and they observed an initial, although not statistically significant, increase in UDVA and CDVA in the first 3 months. However, between the third and sixth postoperative months, their patients showed a gradual return to preoperative functional values associated with a progressive reduction in corneal thickness and the need for re-treatment with epi-off CXL in 50\% of pediatric patients. ${ }^{24}$ These study results therefore suggest that the epi-on technique should not be used in patients under 18 years of age. Magli et al reported the results of a study performed on two groups of patients using both techniques; all patients presented a corneal thickness greater than $400 \mu \mathrm{m}$ and homogeneous topographic and anatomical parameters, but the patients treated were younger than the patients in our study ( $<18$ years) ${ }^{11}$ Both studies deal with the epi-on CXL technique in pediatric patients with discordant results. In our study, no patients needed re-treatment, and in both groups, a significant improvement in visual acuity and topographic parameter was observed. In fact, after treatment (12-month observation time point), $80 \%$ of our patients had a visual acuity greater than $0.2 \operatorname{LogMAR}$, highlighting the effectiveness of the technique epi-on CXL in adult patients.

No complications were recorded in either group. In the literature, several complications have been reported especially after epi-off CXL, such as corneal haze, melting, endothelial damage, and sterile infiltrate infections. ${ }^{28-31}$ In addition, we observed a significantly greater mean postoperative pain in the epi-off CXL group compared to the epi-on CXL group perhaps due to the exposure of corneal nerves and the release of inflammatory mediators, especially prostaglandins and neuropeptides after epithelium removal.

An overall analysis of the clinical outcomes after epi-off and epi-on CXL showed that keratoconus was relatively stable after 12 months, and no differences were observed comparing the two procedures. Despite the different penetration stroma demonstrated in other studies, we observed no clinical differences comparing the two groups. In addition, the risk of infection and pain in the group treated with epi-on CXL was much lower than in the epi-off CXL group. We believe that the lack of a unanimous agreement on the effectiveness of the CXL treatment is due to the presence of intrinsic confounding factors strictly related to the patients. 
In fact, as evidenced by some authors, effectiveness of CXL varies according to age and keratometry. ${ }^{8,32}$

\section{Conclusion}

The current study is the first that describes and compares the results of two CXL techniques performed on two groups of patients with homogeneous clinical and topographical characteristics, highlighting the effectiveness of the epi-on CXL technique in adult patients and proposing it as an alternative to the epi-off CXL. However, our work presents some limitations: the small number of treated eyes and a short follow-up length (12 months). For this reason, our results are not sufficient to assess the differences in efficacy and long-term stability between the two procedures, in particular, epi-on CXL. Further studies with a larger number of eyes and a longer follow-up length are needed to answer these questions.

\section{Acknowledgments}

The authors thank Dr Carmela Acerra (English native speaker) for linguistic revision of the manuscript and Dr Paolo Melillo for statistical analysis.

\section{Disclosure}

The authors report no conflicts of interest in this work.

\section{References}

1. Rabinowitz YS. Keratoconus. Surv Ophthalmol. 1998;42:297-319.

2. Andreassen TT, Simonsen AH, Oxlund H. Biomechanical properties of keratoconus and normal corneas. Exp Eye Res. 1980;31:435-441.

3. Wollensak G, Wilsch M, Spoerl E, Seiler T. Collagen fiber diameter in the rabbit cornea after collagen crosslinking by riboflavin/UVA. Cornea. 2004;23:503-507.

4. Wollensak G, Iomdina E. Biomechanical and histological changes after corneal crosslinking with and without epithelial debridement. $J$ Cataract Refract Surg. 2009;35:540-546.

5. Mencucci R, Marini M, Gheri G, et al. Lectin binding in normal, keratoconus and cross-linked human corneas. Acta Histochem. 2011;113: 308-316.

6. Spoerl E, Wollensak G, Seiler T. Increased resistance of crosslinked cornea against enzymatic digestion. Curr Eye Res. 2004;29:35-40.

7. Vinciguerra P, Albè E, Trazza S, et al. Refractive, topographic, tomographic, and aberrometric analysis of keratoconic eyes undergoing corneal cross-linking. Ophthalmology. 2009;116:369-378.

8. Vinciguerra R, Romano MR, Camesasca FI, et al. Corneal cross-linking as a treatment for keratoconus: four-year morphologic and clinical outcomes with respect to patient age. Ophthalmology. 2013;120: 908-916.

9. O'Brart DP, Kwong TQ, Patel P, McDonald RJ, O'Brart NA. Longterm follow-up of riboflavin/ultraviolet A $(370 \mathrm{~nm})$ corneal collagen cross-linking to halt the progression of keratoconus. Br J Ophthalmol. 2013;97:433-437.

10. Caporossi A, Mazzotta C, Baiocchi S, Caporossi T. Long-term results of riboflavin ultraviolet a corneal collagen cross-linking for keratoconus in Italy: the Siena eye cross study. Am J Ophthalmol. 2010;149:585-593.

11. Magli A, Forte R, Tortori A, Capasso L, Marsico G, Piozzi E. Epithelium-off corneal collagen cross-linking versus transepithelial cross-linking for pediatric keratoconus. Cornea. 2013;32:597-601.
12. Grewal DS, Brar GS, Jain R, Sood V, Singla M, Grewal SP. Corneal collagen crosslinking using riboflavin and ultraviolet-A light for keratoconus: one-year analysis using Scheimpflug imaging. J Cataract Refract Surg. 2009;35:425-456.

13. Filippello M, Stagni E, O'Brart D. Transepithelial corneal collagen crosslinking: bilateral study. J Cataract Refract Surg. 2012;38:283-291.

14. Raiskup-Wolf F, Hoyer A, Spoerl E, Pillunat LE. Collagen crosslinking with riboflavin and ultraviolet-A light in keratoconus: long-term results. $J$ Cataract Refract Surg. 2008;34:796-801.

15. Mazzotta C, Balestrazzi A, Traversi C, et al. Treatment of progressive keratoconus by riboflavin-UVA-induced cross-linking of corneal collagen: ultrastructural analysis by Heidelberg Retinal Tomograph II in vivo confocal microscopy in humans. Cornea. 2007;26:390-397.

16. Mazzotta C, Traversi C, Baiocchi S, et al. Corneal healing after riboflavin ultraviolet-A collagen cross-linking determined by confocal laser scanning microscopy in vivo: early and late modifications. $\mathrm{Am}$ J Ophthalmol. 2008;146:527-533.

17. Mazzotta C, Traversi C, Baiocchi S, Sergio P, Caporossi T, Caporossi A. Conservative treatment of keratoconus by riboflavin-UVA-induced cross-linking of corneal collagen: qualitative investigation. Eur $J$ Ophthalmol. 2006;16:530-535.

18. Caporossi A, Mazzotta C, Baiocchi S, Caporossi T, Paradiso AL. Transepithelial corneal collagen crosslinking for keratoconus: qualitative investigation by in vivo HRT II confocal analysis. Eur J Ophthalmol. 2012;22(suppl 7):S81-S8830.

19. Al-Aqaba M, Calienno R, Fares U, et al. The effect of standard and transepithelial ultraviolet collagen cross-linking on human corneal nerves: an ex vivo study. Am J Ophthalmol. 2012;153:258-266.

20. Xia Y, Chai X, Zhou C, Ren Q. Corneal nerve morphology and sensitivity changes after ultraviolet A/riboflavin treatment. Exp Eye Res. 2011; 93:541-547.

21. Leccisotti A, Islam T. Transepithelial corneal collagen crosslinking in keratoconus. J Refract Surg. 2010;26:942-948.

22. Baiocchi S, Mazzotta C, Cerretani D, Caporossi T, Caporossi A. Corneal crosslinking: riboflavin concentration in corneal stroma exposed with and without epithelium. J Cataract Refract Surg. 2009;35:893-899.

23. Spadea L, Mencucci R. Transepithelial corneal collagen cross-linking in ultrathin keratoconic corneas. Clin Ophthalmol. 2012;6:1785-1792.

24. Caporossi A, Mazzotta C, Paradiso AL, Baiocchi S, Marigliani D, Caporossi T. Transepithelial corneal collagen crosslinking for progressive keratoconus: 24-month clinical results. J Cataract Refract Surg. 2013; 39:1157-1163.

25. Wollensak G, Spoerl E, Seiler T. Riboflavin/ultraviolet-a-induced collagen crosslinking for the treatment of keratoconus. Am J Ophthalmol. 2003;135:620-627.

26. Vinciguerra P, Albe E, Trazza S, Seiler T, Epstein D. Intraoperative and postoperative effects of corneal collagen cross-linking on progressive keratoconus. Arch Ophthalmol. 2009;127:1258-1265.

27. Sharma A, Nottage JM, Mirchia K, Sharma R, Mohan K, Nirankari VS. Persistent corneal edema after collagen cross-linking for keratoconus. Am J Ophthalmol. 2012;154(922-926):e1.

28. Rama P, Di Matteo F, Matuska S, Paganoni G, Spinelli A. Acanthamoeba keratitis with perforation after corneal crosslinking and bandage contact lens use. J Cataract Refract Surg. 2009;35:788-791.

29. Rama P, Di Matteo F, Matuska S, Insacco C, Paganoni G. Severe keratitis following corneal cross-linking for keratoconus. Acta Ophthalmol. 2011;89:e658-e659.

30. Dhawan S, Rao K, Natrajan S. Complications of corneal collagen crosslinking. J Ophthalmol. 2011;2011:869015.

31. Greenstein SA, Hersh PS. Characteristics influencing outcomes of corneal collagen crosslinking for keratoconus and ectasia: implications for patient selection. J Cataract Refract Surg. 2013;39:1133-1140.

32. Wollensak G, Spoerl E, Wilsch M, Seiler T. Keratocyte apoptosis after corneal collagen cross-linking using riboflavin/UVA treatment. Cornea. 2004;23:43-49. 


\section{Publish your work in this journal}

Clinical Ophthalmology is an international, peer-reviewed journal covering all subspecialties within ophthalmology. Key topics include: Optometry; Visual science; Pharmacology and drug therapy in eye diseases; Basic Sciences; Primary and Secondary eye care; Patien Safety and Quality of Care Improvements. This journal is indexed on

Submit your manuscript here: http://www.dovepress.com/clinical-ophthalmology-journal
Dovepress

PubMed Central and CAS, and is the official journal of The Society of Clinical Ophthalmology (SCO). The manuscript management system is completely online and includes a very quick and fair peer-review system, which is all easy to use. Visit http://www.dovepress.com/ testimonials.php to read real quotes from published authors. 